\title{
Jensen-Shannon divergence as a measure of distinguishability between mixed quantum states
}

\author{
A.P. Majtey, ${ }^{1}$ P.W. Lamberti, ${ }^{1}$ and D.P. Prato $^{2}$ \\ ${ }^{1}$ Facultad de Matemática, Astronomía y Física \\ Universidad Nacional de Córdoba \\ Ciudad Universitaria, 5000 Córdoba, Argentina \\ CONICET \\ ${ }^{2}$ Facultad de Matemática, Astronomía y Física \\ Universidad Nacional de Córdoba \\ Ciudad Universitaria, 5000 Córdoba, Argentina
}

(Dated: February 1, 2008)

\begin{abstract}
We discuss an alternative to relative entropy as a measure of distance between mixed quantum states. The proposed quantity is an extension to the realm of quantum theory of the JensenShannon divergence (JSD) between probability distributions. The JSD has several interesting properties. It arises in information theory and, unlike the Kullback-Leibler divergence, it is symmetric, always well defined and bounded. We show that the quantum JSD (QJSD) shares with the relative entropy most of the physically relevant properties, in particular those required for a "good" quantum distinguishability measure. We relate it to other known quantum distances and we suggest possible applications in the field of the quantum information theory.

PACS numbers: 02.50.-r, 03.65.-w, 89.70.+c

Key words: Hilbert space distances, distinguishability distances, Jensen-Shannon divergence, entanglement measures, fidelity.
\end{abstract}




\section{INTRODUCTION}

Distance measures play a central role in quantum theory, in particular in the context of quantum computation and quantum information. On one side they allow us to establish precisely the problem of quantum state discrimination [1]; on the other they are associated to the definition of the degree of entanglement, just to mention two very significant subjects [2].

Several distances between quantum states have been introduced. Many of them were defined as distances (divergences) between probability distributions and then extended as distances between quantum states. This is the case of the Hellinger distance, which, for two (discrete or continuous) probability distributions $P(x)$ and $Q(x)$ reads

$$
H(P, Q)=\sum_{x}[\sqrt{P(x)}-\sqrt{Q(x)}]^{2}
$$

and its quantum mechanical version is expressed as

$$
H(\rho \| \sigma)=\operatorname{Tr}(\sqrt{\rho}-\sqrt{\sigma})^{2}
$$

where $\rho$ and $\sigma$ are two density operators and Tr stands for the trace operator. Analogously the Kullback-Leibler divergence $S(P, Q)=\sum_{x} P(x) \log \left\{\frac{P(x)}{Q(x)}\right\}$ and the Kolmogorov distance, $K(P, Q)=\frac{1}{2} \sum_{x}|P(x)-Q(x)|$ are extended to the realm of quantum mechanics. In the first case the resulting distance is known as the relative entropy [3], [4]; in the second one the quantum counterpart is given by [ $[\underline{5}]$

$$
K(\rho \| \sigma)=\frac{1}{2} \operatorname{Tr}|\rho-\sigma| .
$$

Alternatives to these quantum distances have been recently proposed [6], [7], [8].

As we mentioned previously a basic issue in quantum information theory is to distinguish two quantum states by quantum measurement. In a seminal paper Wootters investigated this problem and introduced a "distinguishability distance" between two pure states [9]. He defines this distance as the number of distinguishable intermediate states between the two states. Braunstein and Caves extended this distance to density operators for mixed states 10] and framed it in a general geometric formulation of quantum states space [1].

In a recent paper we revised the Wootters's distinguishability distance in terms of the Jensen-Shannon divergence (JSD) [12]. The JSD between the probability distributions $P(x)$ 
and $Q(x)$ is defined by:

$$
\begin{aligned}
J S(P, Q) & \equiv S\left(P, \frac{P+Q}{2}\right)+S\left(Q, \frac{P+Q}{2}\right) \\
& =H_{S}\left(\frac{P+Q}{2}\right)-\frac{1}{2} H_{S}(P)-\frac{1}{2} H_{S}(Q)
\end{aligned}
$$

where $S(P, Q)$ is the Kullback-Leibler divergence and $H_{S}(P)=-\sum_{x} P(x) \log P(x)$ is the Shannon entropy. This quantity was introduced by C. Rao [13] and J. Lin [14] as a symmetrized version of the Kullback-Leibler divergence and has been recently applied to the analysis of symbolic sequences as well as to other problems of interest in statistical physics 15]. It originates in information theory, it is always well defined and bounded and its square root is a true metric for the probability distributions space (i.e., its square root is symmetric, null only when the probability distributions coincide and it verifies the triangle inequality) [16]. It has several interesting interpretations; for example in statistical inference theory it gives both the lower and upper bounds to Bayes' probability error [14]. In the framework of information theory the JSD can be related to mutual information [15].

¿From some results of harmonic analysis it is possible to show that the metric space $\left(X_{N}^{+}, \sqrt{J S}\right)$, where $X_{N}^{+}$denotes the discrete probability distributions space, can be isometrically mapped into a subset of a Hilbert space [17], 18]. This result establishes a connection between information theory and differential geometry, which, we think, could have interesting consequences in the realm of quantum information theory.

In reference [14], J. Lin proposed a generalization of Eq. (3) as a distance for several probability distributions. In fact, let $P_{1}(x), \ldots, P_{N}(x)$ be a set of probability distributions and let $\pi_{1}, \ldots, \pi_{N}$ be a collection of non-negative numbers such that $\sum_{i} \pi_{i}=1$. Then the JSD of the probability distributions $P_{i}(x), i=1 \ldots N$ is defined by

$$
J S^{\left(\pi_{1}, \ldots, \pi_{N}\right)}\left(P_{1}, \ldots, P_{N}\right)=H_{S}\left(\sum_{i=1}^{N} \pi_{i} P_{i}\right)-\sum_{i=1}^{N} \pi_{i} H_{S}\left(P_{i}\right)
$$

A remarkable feature of this generalized JSD is that it is possible to assign different weights to the distributions $P_{i}$. This might be of importance in the study of quantum statistical inference problems [19].

In this work we extend the JSD into the context of quantum theory as a distance measure between mixed quantum states. We show that it shares with the relative entropy many of the most physically relevant properties. We also relate it to other commonly used distances 
as well as we suggest its applicability as a good measure of entanglement and fidelity. We see that, when expression (41) is extended to quantum theory, it can be interpreted as the upper bound for quantum accessible information.

The structure of the paper is as follows. In the following section we review the basic properties of the relative entropy, mainly to guide us in our investigation of the properties satisfied by the QJSD. In section III we introduce the QJSD and enumerate its basic properties. Finally we discuss possible applications of the QJSD in the field of quantum information theory.

Notation remark: We will use the following notation: $D($,$) denotes a distance defined$ between probability distributions; $D(\|)$ denotes the corresponding distance between density operators.

\section{RELATIVE ENTROPY}

Let $\mathcal{H}$ be the Hilbert space associated to a quantum system and let $\mathcal{S}$ be the set of density operators describing the states of the system; i.e. $\mathcal{S}$ is the set of self-adjoint, positive and trace unity operators.

The relative entropy of an operator $\rho$, with respect to an operator $\sigma$, both belonging to $\mathcal{S}$, is given by

$$
S(\rho \| \sigma)=\operatorname{Tr}[\rho(\log \rho-\log \sigma)]
$$

where log stands for logarithm in base two. $S(\rho \| \sigma)$ is nonnegative and vanishes if and only if $\rho=\sigma$. It is nonsymmetric and unbounded. In particular, the relative entropy is well defined only when the support of $\sigma$ is equal to or larger than that of $\rho$. Otherwise, it is defined to be $+\infty$ [3]; (the support of an operator is the subspace spanned by the eigenvectors of the operator with non-zero eigenvalues). This is a very restrictive requirement which is violated in some physically relevant situations, for example when $\sigma$ is a pure reference state [8].

Now we list the fundamental properties of the relative entropy. For the proofs and a detailed discussion see references [4] and [20]:

1. $S$ is invariant under unitary transformations, that is

$$
S\left(U \rho U^{\dagger} \| U \sigma U^{\dagger}\right)=S(\rho \| \sigma)
$$


for any unitary operator $U$. This is a quite natural property to be satisfied by a distance, because a unitary transformation represents a rotation in the Hilbert space and the distance between two states should be invariant under a rotation of the states.

2. "Generalized $H$-theorem". For any complete positive, trace preserving map $\Phi$ given by

$$
\begin{gathered}
\Phi \sigma=\sum_{i} V_{i} \sigma V_{i}^{\dagger} \text { and } \sum_{i} V_{i}^{\dagger} V_{i}=1 \\
S(\Phi \rho \| \Phi \sigma) \leq S(\rho \| \sigma)
\end{gathered}
$$

This is a very significant result because the most general way that an open quantum system evolves is mathematically represented by a map of the type given by Eq. (17). Technically, this kind of map is known as a $C P$ - map. Therefore the meaning of Eq. (8) is that non-unitary evolution decreases distinguishability between states. Of course, an unitary evolution is a particular case of a $C P-$ map.

Another example of a $C P-$ map is given by $\sum_{i} P_{i} \sigma P_{i}$ with $P_{i}$ being a complete set of orthogonal projectors $\left(P_{i}^{\dagger}=P_{i}\right.$ and $\left.P_{i}^{2}=P_{i}\right)$. Therefore,

$$
S\left(P_{i} \rho P_{i} \| P_{i} \sigma P_{i}\right) \leq S(\rho \| \sigma)
$$

3. $S$ is jointly convex:

$$
S\left(\sum_{i} \lambda_{i} \rho^{(i)} \| \sum_{i} \lambda_{i} \sigma^{(i)}\right) \leq \sum_{i} \lambda_{i} S\left(\rho^{(i)} \| \sigma^{(i)}\right)
$$

where the $\lambda_{i}$ are positive real numbers such that $\sum_{i} \lambda_{i}=1$.

4. Let $\rho^{A B}$ and $\sigma^{A B}$ be two density matrices of a composite system $A B$ (represented by the Hilbert space $\left.\mathcal{H}_{A} \otimes \mathcal{H}_{B}\right)$. Then

$$
\begin{aligned}
& S\left(\rho^{A} \| \sigma^{A}\right) \leq S\left(\rho^{A B} \| \sigma^{A B}\right) \\
& S\left(\rho^{B} \| \sigma^{B}\right) \leq S\left(\rho^{A B} \| \sigma^{A B}\right)
\end{aligned}
$$

with $\rho^{A}=\operatorname{Tr}_{B} \rho^{A B}$ and $\rho^{B}=\operatorname{Tr}_{A} \rho^{A B}$ (here $\operatorname{Tr}_{A}$ and $\operatorname{Tr}_{B}$ represent the partial trace operators). These inequalities have a very natural interpretation: to take the trace over a part of a system leads to a loss of information and therefore it becomes more difficult to distinguish between two states of the composite system. 
5. $S$ is additive in the sense that

$$
S\left(\rho_{1} \otimes \rho_{2} \| \sigma_{1} \otimes \sigma_{2}\right)=S\left(\rho_{1} \| \sigma_{1}\right)+S\left(\rho_{2} \| \sigma_{2}\right)
$$

with $\rho_{1}, \sigma_{1} \in \mathcal{S}_{A}$ and $\rho_{2}, \sigma_{2} \epsilon \mathcal{S}_{B}$.

6. The relative entropy verifies Donald's identity [21]: Let us suppose that the density operators $\rho_{i}$ occur with probability $p_{i}$, yielding an average state $\rho=\sum_{i} p_{i} \rho_{i}$ and let $\sigma$ be an arbitrary density operator. Then

$$
\sum_{i} p_{i} S\left(\rho_{i} \| \sigma\right)=\sum_{i} p_{i} S\left(\rho_{i} \| \rho\right)+S(\rho \| \sigma)
$$

\section{AN ALTERNATIVE TO THE RELATIVE ENTROPY}

We define the quantum Jensen-Shannon divergence (QJSD) as

$$
J S(\rho \| \sigma)=\frac{1}{2}\left[S\left(\rho \| \frac{\rho+\sigma}{2}\right)+S\left(\sigma \| \frac{\rho+\sigma}{2}\right)\right]
$$

which can be rewritten in terms of the von Neumann entropy, $H_{N}(\rho)=-\operatorname{Tr}(\rho \log \rho)$, as

$$
J S(\rho \| \sigma)=H_{N}\left(\frac{\rho+\sigma}{2}\right)-\frac{1}{2} H_{N}(\rho)-\frac{1}{2} H_{N}(\sigma)
$$

If $\rho$ and $\sigma$ are density operators with complete sets of eigenvectors $\left\{\left|r_{i}\right\rangle\right\}$ and $\left\{\left|s_{i}\right\rangle\right\}$, such that $\rho=\sum_{i} r_{i}\left|r_{i}\right\rangle\left\langle r_{i}\right|$ and $\sigma=\sum_{j} s_{j}\left|s_{j}\right\rangle\left\langle s_{j}\right|$, then the QJSD between $\rho$ and $\sigma$ is expressed as

$$
J S(\rho \| \sigma)=\frac{1}{2}\left\{\sum_{k, i}\left|\left\langle t_{k} \mid r_{i}\right\rangle\right|^{2} r_{i} \log \left(\frac{2 r_{i}}{\lambda_{k}}\right)+\sum_{k, j}\left|\left\langle t_{k} \mid s_{j}\right\rangle\right|^{2} s_{j} \log \left(\frac{2 s_{j}}{\lambda_{k}}\right)\right\}
$$

with $\lambda_{k}=\sum_{i} r_{i}\left|\left\langle t_{k} \mid r_{i}\right\rangle\right|^{2}+\sum_{j} s_{j}\left|\left\langle t_{k} \mid s_{j}\right\rangle\right|^{2}$ and $\left\{\left|t_{k}\right\rangle\right\}$ a complete set of normalized eigenvectors of $\rho+\sigma$.

This quantity is positive, null if and only if $\rho=\sigma$, symmetric and always well defined. In fact, the restriction imposed on the supports of $\rho$ and $\sigma$ for the relative entropy is lifted for the QJSD.

If $\rho$ and $\sigma$ commute they are diagonal in the same basis, that is,

$$
\rho=\sum_{i} r_{i}|i\rangle\left\langle i\left|\quad \sigma=\sum_{i} s_{i}\right| i\right\rangle\langle i|
$$

with $|i\rangle$ an orthonormal basis. Then

$$
J S(\rho \| \sigma)=J S\left(\left\{r_{i}\right\},\left\{s_{i}\right\}\right)
$$


The QJSD is bounded. In fact, as it is known, the von Neumann entropy satisfies the following inequality [1]: if $\rho=\sum_{i} p_{i} \rho_{i}$ is a mixture of quantum states $\rho_{i}$ with $p_{i}$ a set of positive real numbers such that $\sum_{i} p_{i}=1$, then

$$
H_{N}\left(\sum_{i} p_{i} \rho_{i}\right) \leq \sum_{i} p_{i} H_{N}\left(\rho_{i}\right)+H_{S}\left(\left\{p_{i}\right\}\right)
$$

The equality is attained if and only if the states $\rho_{i}$ have support on orthogonal subspaces. By putting $p_{1}=p_{2}=\frac{1}{2}$ and $\rho_{1}=\rho$ and $\rho_{2}=\sigma$ in the inequality above we have

$$
0 \leq J S(\rho \| \sigma) \leq 1
$$

Properties 1 to 4 of relative entropy are inherited by the QJSD and their validity can be checked directly from the representation (14). For example, property 2 for the QJSD can be proved as follows:

$$
\begin{aligned}
J S(\Phi \rho \| \Phi \sigma) & =\frac{1}{2}\left[S\left(\Phi \rho \| \frac{\Phi \rho+\Phi \sigma}{2}\right)+S\left(\Phi \sigma \| \frac{\Phi \rho+\Phi \sigma}{2}\right)\right] \\
& =\frac{1}{2}\left[S\left(\Phi \rho \| \frac{\Phi(\rho+\sigma)}{2}\right)+S\left(\Phi \sigma \mid \frac{\Phi(\rho+\sigma)}{2}\right)\right] \\
& \leq \frac{1}{2}\left[S\left(\rho \| \frac{\rho+\sigma}{2}\right)+S\left(\sigma \| \frac{\rho+\sigma}{2}\right)\right]=J S(\rho \| \sigma)
\end{aligned}
$$

Additivity does not remain valid for the QJSD. However, the QJSD verifies a "restricted additivity":

$$
J S\left(\rho_{1} \otimes \rho_{2} \| \sigma_{1} \otimes \rho_{2}\right)=J S\left(\rho_{1} \| \sigma_{1}\right)
$$

with $\rho_{1}, \sigma_{1} \in \mathcal{S}_{A}$ and $\rho_{2} \epsilon \mathcal{S}_{B}$. This is an important point that deserves some attention. We claim that (21) is enough to prove property 4 for the QJSD. To prove this, we follow the same steps as Nielsen and Chuang in their book where the relative entropy is studied [1]. It can be shown that there are unitary transformations $U_{j}$ on the space corresponding to the part $B$ and numbers $\lambda_{j},\left(\sum_{j} \lambda_{j}=1\right)$ such that

$$
\rho^{A} \otimes \frac{I}{d}=\sum_{j} \lambda_{j} U_{j} \rho^{A B} U_{j}^{\dagger}
$$

for all density operator $\rho^{A B}$ of the composite system $A B$. Here $d$ is the dimension of the Hilbert space of part $B$ and $I$ its identity operator. Hence, from convexity, the invariance under unitary evolution and the restricted additivity verified by the QJSD, we have:

$$
J S\left(\rho^{A} \| \sigma^{A}\right)=J S\left(\rho^{A} \otimes \frac{I}{d} \| \sigma^{A} \otimes \frac{I}{d}\right)
$$




$$
\begin{aligned}
& \leq \sum_{j} \lambda_{j} J S\left(U_{j} \rho^{A B} U_{j}^{\dagger} \| U_{j} \sigma^{A B} U_{j}^{\dagger}\right) \\
& \leq \sum_{j} \lambda_{j} J S\left(\rho^{A B} \| \sigma^{A B}\right)=J S\left(\rho^{A B} \| \sigma^{A B}\right)
\end{aligned}
$$

Of course, due to the linearity of the partial trace operator, this property can be derived from the analogous ones satisfied by the relative entropy.

The non-increasing character of the QJSD upon a $C P$ - map has the following consequence: Let $\left\{E_{\alpha}\right\}$ be a set of positive - operator - valued measure (POVM), that is, a set of Hermitian operators $E_{\alpha} \geq 0$ that satisfy $\sum_{\alpha} E_{\alpha}=1$. It is known that every generalized quantum measurement can be described by a POVM [1]. Given two operators $\rho$ and $\sigma$ belonging to $\mathcal{S}$, we can introduce the probability distributions

$$
p_{\alpha}=\operatorname{Tr}\left(\rho E_{\alpha}\right) \quad q_{\alpha}=\operatorname{Tr}\left(\sigma E_{\alpha}\right)
$$

Following Vedral [20] we define

$$
J S_{1}(\rho \| \sigma)=\sup _{\left\{E_{\alpha}\right\}} J S\left(\left\{p_{\alpha}\right\},\left\{q_{\alpha}\right\}\right)
$$

where the supremum is taken over all POVM's. Given that $S(\rho \| \sigma) \geq S_{1}(\rho \| \sigma)$ with $S_{1}(\rho \| \sigma)=\sup _{\left\{E_{\alpha}\right\}} S\left(\left\{p_{\alpha}\right\},\left\{q_{\alpha}\right\}\right)$, we can derive the inequality

$$
J S(\rho \| \sigma) \geq J S_{1}\left(\left\{p_{\alpha}\right\},\left\{q_{\alpha}\right\}\right)
$$

Of course the map $\rho \rightarrow \operatorname{Tr}\left(\rho E_{\alpha}\right)$ is a $C P-$ map.

To conclude the listing of properties satisfied by the QJSD we note that starting from Donald's identity, we can obtain a useful representation for the QJSD:

$$
2 J S(\rho \| \sigma)=S(\rho \| \tau)+S(\sigma \| \tau)-2 S\left(\frac{\rho+\sigma}{2} \| \tau\right)
$$

where $\tau$ is an arbitrary density operator. This identity allows us to evaluate the QJSD between two density operators in terms of a third arbitrary one.

In the following discussion we investigate the relation between the QJSD and other quantum distances. In reference 12] we showed that the JSD is a good approximation to the Wootters distance (up to third order in their power expansion). To search for a similar relationship between the QJSD distance and that introduced by Braunstein and Caves, let us 
consider two neighboring density operators, $\rho$ and $\rho+d \rho$. The QJSD between them reduces to (up to second order in $\varepsilon$ )

$$
J S(\rho \| \rho+d \rho)=\frac{\varepsilon^{2}}{8}\left[\sum_{j} \frac{\omega_{j j}}{p_{j}}+\sum_{j, k}\left(p_{j}-p_{k}\right)\left(\log p_{j}-\log p_{k}\right)\left|a_{k j}\right|^{2}\right]
$$

where we have assumed that $d \rho \equiv \varepsilon \omega$, with $\varepsilon \ll 1$. The $\omega_{j k}$ are the matrix elements of $\omega$ in the basis that diagonalizes $\rho$, that is $\rho=\sum_{j} p_{j}|j\rangle\langle j|$, and the eigenvectors of $\rho+d \rho$ are written (up to first order in $\varepsilon$ ) in the form:

$$
\left|j^{(1)}\right\rangle=\sum_{k}\left(\delta_{k j}+\varepsilon a_{j k}\right)|k\rangle
$$

By expanding $\log p_{j}-\log p_{k}=\frac{2\left(p_{j}-p_{k}\right)}{p_{j}+p_{k}}-\frac{4\left(p_{j}-p_{k}\right)^{3}}{\left(p_{j}+p_{k}\right)^{3}}+\ldots$ and after some algebra, the expression (25) can be rewritten as

$$
J S(\rho \| \rho+d \rho)=\frac{\varepsilon^{2}}{8}\left[\sum_{j} \frac{\omega_{j j}}{p_{j}}+2 \sum_{j, k} \frac{\left(p_{j}-p_{k}\right)^{2}}{p_{k}+p_{j}}\left|a_{k j}\right|^{2}\right]+\vartheta
$$

with $\vartheta$ a term that involves sums of powers of $\left(p_{j}-p_{k}\right)$ of even order not lower than four. The two first terms coincide with the metric introduced by Braunstein and Caves as a generalization of the Wootters's distance, $d s_{D O}[10]$ :

$$
J S(\rho \| \rho+d \rho) \approx \frac{1}{8} d s_{D O}^{2}
$$

As these authors further note, the metric $d s_{D O}$ is related to the Bures' metric, $B(\rho \| \sigma)=$ $\sqrt{ } 2\left[\left(1-\operatorname{Tr}\left(\rho^{1 / 2} \sigma \rho^{1 / 2}\right)^{1 / 2}\right]^{1 / 2}:\right.$

$$
\left.4 B^{2}(\rho \| \rho+d \rho)\right) \approx d s_{D O}^{2}
$$

Therefore, we can conclude that for two neighboring states

$$
B(\rho \| \rho+d \rho) \approx \sqrt{2 J S(\rho \| \rho+d \rho)}
$$

To finish this section, we proceed to evaluate the QJSD in a particular case. Let us consider the density operator $\rho_{W}$ corresponding to a Werner state, for a system of two 1/2-spin particles [22]:

$$
\rho_{W}=F\left|\Psi^{-}\right\rangle\left\langle\Psi^{-}\right|+\frac{1-F}{3}\left(\left|\Psi^{+}\right\rangle\left\langle\Psi^{+}|+| \Phi^{+}\right\rangle\left\langle\Phi^{+}|+| \Phi^{-}\right\rangle\left\langle\Phi^{-}\right|\right)
$$


with $\left|\Psi^{ \pm}\right\rangle=\frac{1}{\sqrt{ } 2}(|\uparrow \downarrow\rangle \pm|\downarrow \uparrow\rangle)$ and $\left|\Phi^{ \pm}\right\rangle=\frac{1}{\sqrt{ } 2}(|\uparrow \uparrow\rangle \pm|\downarrow \downarrow\rangle)$. $F$ is the purity of the state $\rho_{W}$ with respect to the reference state $\sigma=\left|\Psi^{-}\right\rangle\left\langle\Psi^{-}\right|$. Then, in terms of the purity $F$, the QJSD between $\rho_{W}$ and $\sigma$, results:

$$
J S\left(\rho_{W} \| \sigma\right)=\frac{1}{2}\left[F \log F-(1+F) \log \left(\frac{1+F}{2}\right)\right]
$$

The fact that we can evaluate the $J S(\rho \| \sigma)$ when one of its arguments is a pure state, is a clear advantage of the QJSD over the relative entropy, which becomes divergent in this case.

\section{DISCUSSION}

In this section we suggest possible applications of the QJSD in the context of quantum information theory. Here we show that the proposed distance is useful to generate an entanglement measure, as well as an alternative concept of fidelity. We also give an interesting interpretation of QJSD as the upper bound for the accessible quantum information. A more detailed study of each one of these proposals will be presented in a future work.

\section{A. Entanglement}

Let $\mathcal{H}=\mathcal{H}_{\mathcal{A}} \otimes \mathcal{H}_{\mathcal{B}}$ be the Hilbert space of a quantum system consisting of two subsystems $A$ and $B$. According to Vedral et. al. any measure of entanglement $\mathcal{E}$ has to satisfy the following (necessary) conditions [2]:

- i) $\mathcal{E}(\rho)=0$ iff $\rho$ is separable. Let us recall that a state is separable if it can be expressed as $\rho=\sum_{j} \lambda_{j} \rho_{1}^{(j)} \otimes \rho_{2}^{(j)}$ for some density operators $\rho_{1}^{(j)} \epsilon \mathcal{S}_{\mathcal{A}}$ and $\rho_{2}^{(j)} \epsilon \mathcal{S}_{\mathcal{B}}$, and $\sum_{j} \lambda_{j}=1, \lambda_{j} \geq 0$.

- ii) $\mathcal{E}(\rho)=\mathcal{E}\left(U_{A} \otimes U_{B} \rho U_{A}^{\dagger} \otimes U_{B}^{\dagger}\right)$ for two arbitrary unitary operators $U_{A}$ and $U_{B}$ acting on the corresponding Hilbert space.

- iii) $\mathcal{E}(\rho)$ is convex with respect to its argument:

$$
\mathcal{E}\left(\sum_{j} \lambda_{j} \rho_{j}\right) \leq \sum_{j} \lambda_{j} \mathcal{E}\left(\rho_{j}\right)
$$


- iv) $\mathcal{E}(\rho)$ decreases under generic quantum operations, that is, if $\left\{V_{i}\right\}$ are bounded operators such that $\sum V_{i} V_{i}^{\dagger}=1, \rho_{j}=\frac{V_{j} \rho V_{j}^{\dagger}}{\lambda_{j}}$ and $\lambda_{j}=\operatorname{Tr}\left(V_{j} \rho V_{j}^{\dagger}\right)$, then

$$
\mathcal{E}(\rho) \geq \sum_{j} \lambda_{j} \mathcal{E}\left(\rho_{j}\right)
$$

Vedral and collaborators 2] showed that an entanglement measure defined as

$$
\mathcal{E}(\rho)=\inf _{\sigma} D(\rho \| \sigma)
$$

satisfies conditions i)-iv), whenever the distance $D: \mathcal{S} \otimes \mathcal{S} \rightarrow \Re$, verifies the properties a)-b) listed below:

- a) $D(\rho \| \sigma) \geq 0$ and $D(\rho \| \rho)=0$ for any $\rho, \sigma \epsilon \mathcal{S}$

- b) $D(\Phi \rho \| \Phi \sigma) \leq D(\rho \| \sigma)$ for any $C P-\operatorname{map}, \Phi$.

In (29) the infimum is taken over the set of separable states.

As we showed in the preceding section, the QJSD $J S(\rho \| \sigma)$ satisfies these two requirements. So we can introduce a new entanglement measure by

$$
\mathcal{E}_{J S}(\rho)=\inf _{\sigma} J S(\rho \| \sigma)
$$

where, once again, the infimum is taken over all separable states.

\section{B. Fidelity}

The fidelity of states $\rho$ and $\sigma$ is defined as [23], [24]

$$
F(\rho \| \sigma)=(\operatorname{Tr} \sqrt{\sqrt{\rho} \sigma \sqrt{\rho}})^{2}
$$

This quantity is symmetric, invariant under unitary transformations and bounded between 0 and 1 . For a pure state $\sigma=|\Psi\rangle\langle\Psi|$, fidelity reduces to the amplitude $\langle\Psi|\rho| \Psi\rangle$.

The fidelity is related to the Bures's metric by the expression

$$
B^{2}(\rho \| \sigma)=2(1-\sqrt{F(\rho \| \sigma)})
$$

Taking Eq. (27) into consideration we can introduce an alternative definition of fidelity (31):

$$
F_{J S}(\rho \| \sigma) \equiv(1-J S(\rho \| \sigma))^{2}
$$


¿From inequalities (19),$F_{J S}$ must be bounded between 0 and 1. In particular, for $\rho=$ $\sigma, F_{J S}=1$ and $F_{J S}(\rho \| \sigma)=0$ if and only if $\rho$ and $\sigma$ have support on orthogonal subspaces.

The proposal (33) has another justification. As a textbook exercise it can be shown that the fidelity of states $\rho$ and $\sigma$ can be evaluated in terms of purifications of these states as

$$
F(\rho \| \sigma)=\max _{|\varphi\rangle}|\langle\psi \mid \varphi\rangle|^{2}
$$

where $|\psi\rangle$ is any fixed purification of $\rho$, and the maximization is over all the purifications of $\sigma$ 1]. At this point, it should be recalled that the Wootters' distance between the two pure states $|\psi\rangle$ and $|\varphi\rangle$ is given by $[9]$ :

$$
W(|\psi\rangle \||\varphi\rangle)=\arccos |\langle\psi \mid \varphi\rangle|
$$

Then, fidelity can be expressed in terms of the Wootters' distance as

$$
\begin{aligned}
F(\rho \| \sigma) & =\max _{|\varphi\rangle} \cos ^{2}(W(|\psi\rangle \||\varphi\rangle)) \\
& \left.=\max _{|\varphi\rangle}\left(1-W^{2}(|\psi\rangle \||\varphi\rangle)\right)+\ldots\right)
\end{aligned}
$$

Now taking into account the relation between the Wootters's distance and the JSD divergence [12]

$$
W(|\psi\rangle \||\varphi\rangle) \approx \sqrt{2 J S(|\psi\rangle|\|| \varphi\rangle)}
$$

we can approximate

$$
F(\rho \| \sigma) \approx \max _{|\varphi\rangle}(1-J S(|\psi\rangle \||\varphi\rangle))^{2}
$$

which, we think, might be taken as an interesting starting point to investigate (33) as a fidelity measure.

\section{Quantum information accessibility}

Holevo [25] proved that, if a system is prepared in a state $X$ chosen from one of the density operators $\rho_{i},(i=1, \ldots, n)$ with probability $p_{i}$, then the (mutual) information $I(X: Y)$ that can be gathered about the identity of the state $X$ by a POVM measurement, with outcome $Y$, is bounded according to the inequality

$$
I(X: Y) \leq \chi=H_{N}\left(\sum_{i} p_{i} \rho_{i}\right)-\sum_{i} p_{i} H_{N}\left(\rho_{i}\right)
$$


The quantity $\chi$ is precisely the extension of expression (44) to density operators with the weights $\pi_{i}$ replaced by the probabilities $p_{i}$, that is, $\chi \equiv J S^{\left(p_{1}, \ldots p_{n}\right)}\left(\rho_{1}\|\ldots\| \rho_{n}\right)$. This fact provides an interesting interpretation of the generalized QJSD.

\section{AKNOWLEDGMENT}

We are grateful to Secretaria de Ciencia y Tecnica de la Universidad Nacional de Córdoba for financial assistance. This work was partially supported by Grant BIO2002-04014-C03-03 from the Spanish Government.

[1] M.A. Nielsen and I. L. Chuang "Quantum Computation and Quantum Information", Cambridge University Press, Cambridge (2000).

[2] V. Vedral, M. Plenio, M.A. Rippin, and P. Knight, Phys. Rev. Lett. 78, 2275 (1997).

[3] G. Lindblad, Comm. Math. Phys. 33, 305 (1973).

[4] A. Wehrl, Rev. Mod. Phys. 50 (2), 221-260 (1978).

[5] C. Fuchs and J. van de Graaf, IEEE Trans. Inf. Theory, 45, 1216-1227 (1999).

[6] S. Luo and Q. Zhang, Phys. Rev. A, 69, 032106 (2004).

[7] J. Lee and M.S. Kim, Phys. Rev. Lett., 91, 087902 (2003).

[8] S. Abe, Phys. Rev. A, 68, 032302 (2003)

[9] W. Wootters, Phys. Rev D 23, 357 (1981).

[10] S. Braunstein and C. Caves, Phys. Rev. Lett. 723439 (1994).

[11] S. Braunstein and C. Caves, in "Fundamental problems in quantum theory", Edited by D. Greenberger and A. Zeilinger, Annals of the New York Academy of Sciences, 755, 786-797 (1995).

[12] A. Majtey, P.W. Lamberti, M.T. Martin and A. Plastino, Eur. Phys. J. D 32, 413-419 (2005).

[13] C. Rao, "Differential Geometry in Statistical Inference", IMS-Lectures Notes, 10, 217 (1987).

[14] J. Lin, IEEE Trans. Inf. Theory 37,1, 145 (1991).

[15] I. Grosse, P. Bernaola-Galvan, P. Carpena, R. Roman Roldan, J. Oliver y H.E. Stanley, Phys. Rev. E, 65041905 (2002).

[16] D. Endres and J. Schindelin, IEEE Trans. Inf. Theory, 49, 7, 1858 (2003).

[17] I.J. Schoenberg, Trans. Am. Math. Soc. 44, 3 (1938). 
[18] C. Berg, J. Christensen and P. Ressel, Harmonic Analysis on Semigroups, Springer-Verlag, New York (1984).

[19] D. Brady and L. Hughston, Geometric Issues on the Foundations of Science. Eds. S.A. Huggett, L. Mason, K. Tod, S.-T. Tsou and N. Woodhouse, Oxford University Press.

[20] V. Vedral, Rev. Mod. Phys. 74, 197-234 (2002).

[21] M.J. Donald, Math. Proc. Cam. Phil. Soc. 101, 363 (1987).

[22] R.F. Werner, Phys. Rev. A, 40, 4277 (1989).

[23] B. Schumacher, Phys. Rev. A, 51, 2738 (1995).

[24] C. Bennett, G. Brassard, S. Popescu, B. Schumcher, J. Smolin and W. Wootters, Phys. Rev. Lett. 76, 722-725.

[25] A.S. Holevo, Probl. Inform. Transmission 9, 177 (1973). 